\title{
Broadband Fiber-based Entangled Photon-Pair Source at Telecom O-band
}

\author{
Chanjia Chen ${ }^{1 *}$, Calvin Xu${ }^{1}$, Arash Riazi ${ }^{1}$, Eric Y. Zhu ${ }^{1}$, Alexey V.Gladyshev², \\ Peter G.Kazansky³, and Li Qian'
}

\author{
${ }^{1}$ Dept. of Electrical and Computer Engineering, University of Toronto, 10 King's College Rd., Toronto, M5S 3G4, Canada \\ ${ }^{2}$ Prokhorov General Physics Institute of the Russian Academy of Sciences, Dianov Fiber Optics Research Center, 38 Vavilov Street, 119333 Moscow, \\ Russia ${ }^{3}$ Optoelectronics Research Centre, University of Southampton, Southampton SO17 1BJ, United Kingdom \\ *Corresponding author: changjia.chen@mail.utoronto.ca
}

In this letter, we report a polarization-entangled photon-pair source based on type-II spontaneous parametric down conversion at telecom $\mathrm{O}$-band in periodically poled silica fiber (PPSF). The photon-pair source exhibits more than $130 \mathrm{~nm}$ ( $\sim 24 \mathrm{THz})$ emission bandwidth centered at $1306.6 \mathrm{~nm}$. The broad emission spectrum results in a short biphoton correlation time and we experimentally demonstrate a Hong-Ou-Mandel interference dip with a full width of 26.6 fs at half maximum. Owing to the low birefringence of the PPSF, the biphotons generated from type-II SPDC are polarization-entangled over the entire emission bandwidth, with a measured fidelity to a maximally entangled state greater than $95.4 \%$. The biphoton source provides the broadest bandwidth entangled biphotons at O-band to our knowledge.

In the last few decades, the development of photon-pair sources has laid the foundation for quantum photonic technologies. One of the most convenient methods of producing entangled photon-pair is spontaneous parametric downconversion (SPDC) in a second-order nonlinear medium. SPDC has been extensively studied for its applications in quantum information processing and photonic quantum computation[1]. By engineering the nonlinear medium, the photon-pairs generated by SPDC can be naturally endowed with nonclassical correlations, such as entanglement in polarization[2] or in orbital angular momentum[3] degrees of freedom. The frequency-time correlated biphotons in SPDC source are also of broad interest, as they enable quantum interferometry that surpasses the limits of classical sensitivity[4].

In particular, broad bandwidth and time-correlated photonpairs are desirable as they enable the investigation of the optical properties of a sample over a wide spectral range, enhancing the spatial and temporal resolution of quantum sensing[5]. Broadband photon-pair sources are also significant for quantum communication techniques, such as wavelength-multiplexed entanglement distribution[6] and clock synchronization[7]. For example, it is crucial to use broadband time-correlated photonpairs for distant clock synchronization, as its precision is limited by the biphoton correlation time[7]. However, in fiber-based quantum communication where the biphotons are transmitted through long-distance fiber links, precise dispersion compensation is needed because the fiber chromatic dispersion will obscure the timing correlation. A biphoton source centered near the zero-dispersion wavelength (around $1310 \mathrm{~nm}$ ) of the telecom silica fiber may resolve this problem and improve the precision of timing[8]. Various entangled photon sources at telecom Oband (1260 - $1360 \mathrm{~nm})$ with a biphoton emission bandwidth up to a few tens of nanometers have been developed[9-11]. However, this bandwidth is still lacking. Generating broadband and highly time-correlated photon pairs via the process of SPDC remains a challenge. In order to use SPDC to generate broadband, time-correlated biphotons, various methods have been employed, such as using a short nonlinear medium[6] and chirped quasi-phase-matching[12], though at the expense of reduced biphoton generation rate and complex fabrication requirement. A time-correlated photon-pair source at O-band which has broader bandwidth and is compatible with telecom fibers is still highly desired.

In addition to the time-frequency degree of freedom, the polarization correlation within the biphotons generated via SPDC also attracts much interest. Broadband biphotons with polarization entanglement can be used for polarization sensitive quantum sensing[13] and multi-user quantum communication[14]. Yet generating polarization entangled photons over a broad bandwidth often requires stable compensation for the wavelength-dependent birefringence of the nonlinear medium[6, 15]. Interferometric methods or compensating components are therefore needed to achieve a high polarization entanglement quality[16], increasing the experimental difficulties.

A promising solution is to use a nonlinear medium of sufficient interaction length but low birefringence and low chromatic dispersion to generate broadband entangled photon-pairs. In this letter, we demonstrate a cw-pumped periodically poled silica fiber (PPSF) based entangled photon-pair source quasi-phasematched (QPM) at $1306.6 \mathrm{~nm}$, which is near the zero-dispersion wavelength of telecom single-mode silica fiber (e.g. SMF-28). 
The low chromatic dispersion and the low birefringence of the PPSF at telecom O-band result in a biphoton emission bandwidth greater than $130 \mathrm{~nm}$ ( $>24 \mathrm{THz}$ in frequency), which to our knowledge is the broadest bandwidth of polarization-entangled biphotons at O-band. We demonstrate Hong-Ou-Mandel interference with the biphotons source and measure an interference dip width of $26.6 \pm 1.3 \mathrm{fs}$. We also perform quantum state tomography to measure the quality of polarisation entanglement. The concurrence[17] of the polarization entanglement is measured to be more than 0.91 and the fidelity to a maximally entangled state is measured to be more than $95.4 \%$. This broadband PPSF source employs mature telecommunication components and eliminates beam alignment. It is compatible with the existing low-loss telecom fiber links and facilitates long-distance entanglement distribution and compensation-free quantum clock synchronization.

To see how birefringence and chromatic dispersion affect the SPDC spectrum, we may write the spectral brightness of an SPDC source as[18]:

$$
I\left(\omega_{s}, \omega_{i}\right) \propto \operatorname{sinc}^{2}\left[\frac{L}{2}\left(k_{A}\left(\omega_{p}\right)-k_{B}\left(\omega_{s}\right)-k_{C}\left(\omega_{i}\right)-\frac{2 \pi}{\Lambda}\right)\right],
$$

where $k$ is the wavenumber of the PPSF, $L$ is the length of the PPSF, subscripts $p, s, i$ denote pump, signal and idler respectively and $\Lambda$ is the QPM period. The angular frequencies of photons obey energy conservation: $\omega_{s}+\omega_{i}=\omega_{p}$ and we assume $\omega_{i}>$ $\omega_{s}$. Subscripts $A, B$ and $C$ represent the polarization modes of the photons. In the context of this letter, we discuss the type-II SPDC in PPSF, in which a pair of orthogonally polarized photons are generated by the down-conversion of a vertically polarized pump photon, i.e. $A=V[19]$. Therefore, in type-II SPDC, subscripts $B, C$ can be $H, V$ or $V, H$, where $H$ is for horizontally polarized photons and $V$ is for vertically polarized photons. We assume the PPSF is cw-pumped and $k_{A}\left(\omega_{p}\right)=k_{V}\left(\omega_{p}\right) \equiv k_{p}$, thus the QPM condition becomes:

$$
k_{p}-k_{H}\left(\omega_{p} / 2\right)-k_{V}\left(\omega_{p} / 2\right)-\frac{2 \pi}{\Lambda}=0 .
$$

Taylor-expanding the wavenumbers $k(\omega)$ in Eq. (1) about $\omega_{p} / 2$ up to the second-order, and substituting Eq. (2) into Eq. (1) to eliminate the zeroth order terms, we obtain[20]:

$$
I(\Delta) \propto \operatorname{sinc}^{2}\left[\frac{1}{2} M L \Delta+\frac{1}{2} k_{2} L \Delta^{2}+O\left(\Delta^{3}\right)\right],
$$

where $\Delta=\omega_{p} / 2-\omega_{s}=\omega_{i}-\omega_{p} / 2, k_{2}=\partial^{2} k(\omega) /\left.\partial \omega^{2}\right|_{\omega=\omega_{p} / 2}$ is the chromatic dispersion at frequency $\omega_{p} / 2$, and $M=$ $\left.\left(\partial k_{H}(\omega) / \partial \omega-\partial k_{V}(\omega) / \partial \omega\right)\right|_{\omega=\omega_{p} / 2}$ is the group velocity mismatch between $\mathrm{H}$ and $\mathrm{V}$ polarizations. The width of $I(\Delta)$ has an inverse relation to $M$ and $k_{2}$. If $M$ and $k_{2}$ are small enough, the spectral bandwidth of SPDC can be very broad. In general, the SPDC bandwidth in second-order nonlinear crystals such as lithium niobate and potassium titanyl phosphate is significantly limited by their high group birefringence $M$ and nontrivial chromatic dispersion $k_{2}$. On the other hand, the group birefringence $M$ in PPSF has been shown to be negligible[20] and the polarization entanglement of the biphotons in PPSF can be naturally generated without the need of any birefringence compensation[21]. As a result, the bandwidth of the biphotons in PPSF sources is mainly determined by its chromatic dispersion $k_{2}$, and $\Delta \propto 1 / \sqrt{k_{2} L}$. Taking advantage of its low dispersion at
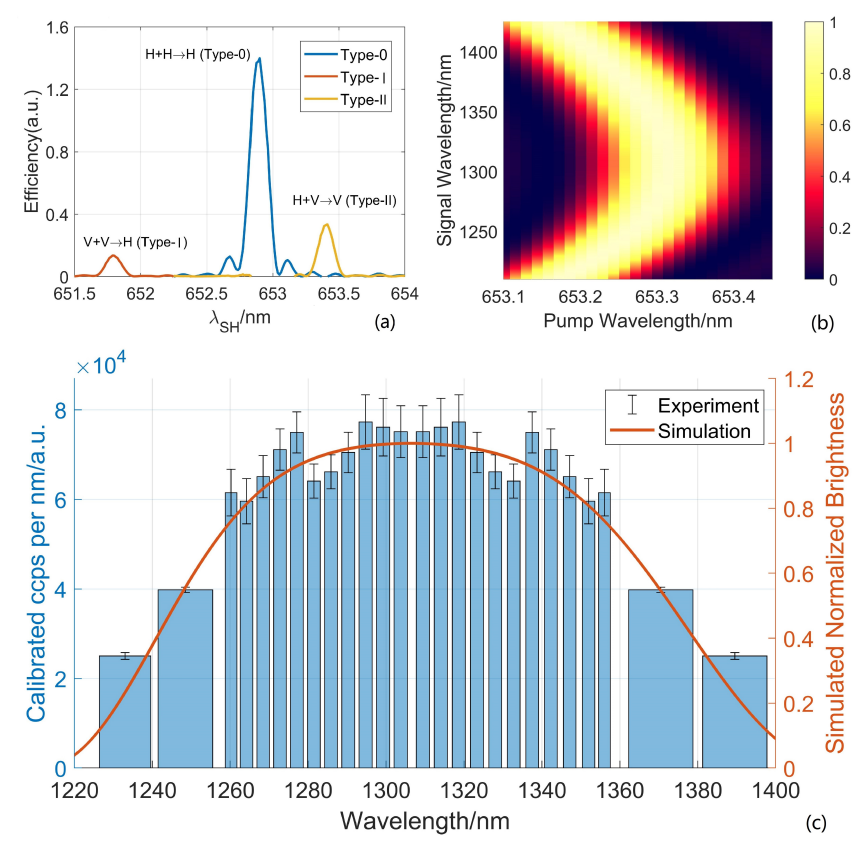

Fig. 1. (a) Experimentally measured SHG spectrum of the PPSF. The weak birefringence in PPSF results in the spectral separation of three polarization-dependent phase-matchings. (b) Theoretical tuning curve of the type-II SPDC emission spectrum in PPSF with respect to the pump wavelength. (c) Experimentally measured biphoton spectrum shows a biphoton emission bandwidth greater than $130 \mathrm{~nm}$ (>24 THz).

O-band, the PPSF is able to generate entangled photons with very broad bandwidth.

The PPSF we use in the source is a 20-cm-long, weakly birefringent step-index silica fiber with an NA $\approx 0.14$ (at the wavelength of $1.55 \mu \mathrm{m}$ ), which is compatible with telecom fibers such as SMF-28[22]. It exhibits second-order nonlinearity which is induced through thermal poling[23]. The QPM condition is achieved through periodic UV erasure with $\Lambda=54 \mu \mathrm{m}$. As shown by the experimentally measured second harmonic generation (SHG) spectrum in Fig.1(a), the PPSF supports three types of SHG, and correspondingly three types of SPDC[19]. Among the three types, the type-II SPDC in PPSF can be used for compensation-free broadband polarization entanglement generation[20]. A finite element eigenmode solver (Lumerical Inc.) is used for calculating the birefringence and dispersion of the fiber modes based on the PPSF's geometry. Assuming a cw-pump is used, we simulate the type-II SPDC spectrum and obtain the tuning curve as a function of the pump wavelength, as shown in Fig.1(b). When the pump wavelength for SPDC is set at around the type-II SHG peak $(653.3 \mathrm{~nm})$, the PPSF can generate broadband biphotons with more than $130 \mathrm{~nm}$ bandwidth.

The biphoton spectrum of type-II SPDC in PPSF is experimentally measured with a pump laser at $653.3 \mathrm{~nm}$ at room temperature $\left(\sim 22^{\circ} \mathrm{C}\right)$, as shown in Fig.1(c). To measure the biphoton spectrum, we first use a pair of fine tunable filters at O-band (O/E land Inc., tuning range $1260 \mathrm{~nm}-1360 \mathrm{~nm}$ ). Outside the tuning range of the fine tunable filters, we use two coarse wavelength division multiplexers (CWDMs, each has a $3 \mathrm{~dB}$ transmission bandwidth of $17 \mathrm{~nm}$ ) centered at $1370 \mathrm{~nm}$ and 1390 $\mathrm{nm}$ respectively. After calibrating the wavelength-dependent 

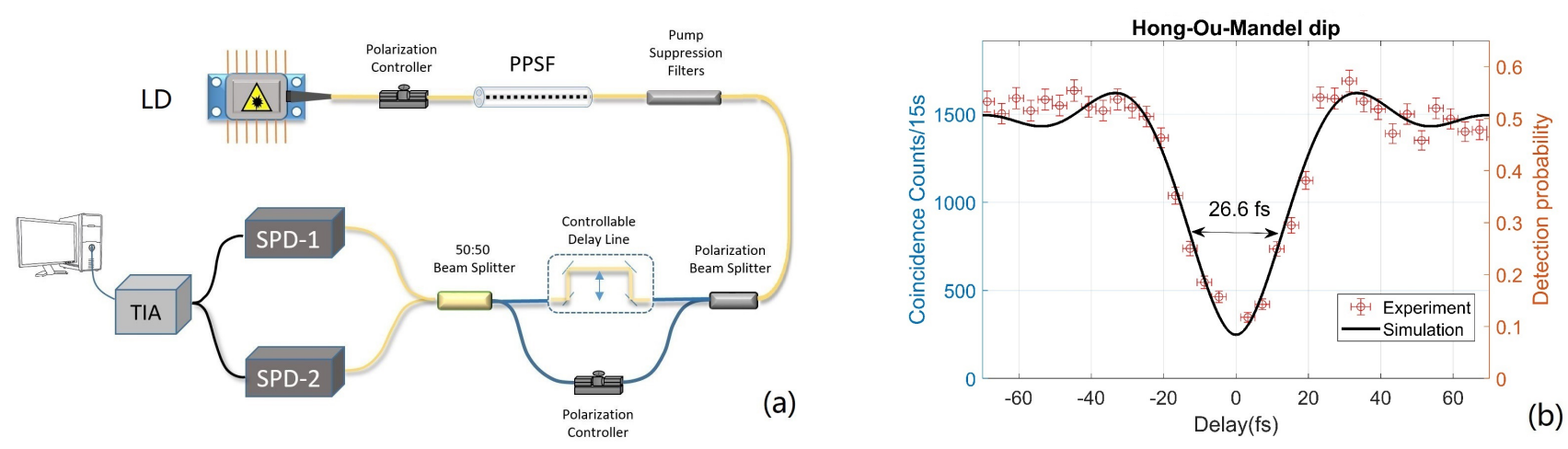

Fig. 2. (a) Experimental setup for the Hong-Ou-Mandel interference Measurement. LD: $\mathrm{cw}$ laser diode with emission wavelength at $653 \mathrm{~nm}$. SPD, single photon detector (IDQ220, ID Quantique, quantum efficiency $\sim 20 \%$ in O-band); TIA, time interval analyzer (Hydraharp 400). (c) Hong-Ou-Mandel interference shows a dip of $83.2 \pm 0.3 \%$ visibility with width of $26.6 \pm 1.3 \mathrm{fs}$.

loss and the detector efficiencies, a spectral bandwidth of more than $130 \mathrm{~nm}$ ( $>24 \mathrm{THz}$ in frequency) of type-II SPDC is obtained from the measured coincidence counts.

In addition to the direct spectral brightness measurement, a Hong-Ou-Mandel interference (HOMI) experiment is also performed as one may use the temporal correlation[24] of the biphotons to infer their bandwidth. The HOMI experimental setup is shown in Fig.2(a). A laser diode that has its wavelength stabilized at $653.3 \mathrm{~nm}$ by a fiber Bragg grating is used as the pump, with its polarization adjusted to align to the slow axis (defined as the V polarization in PPSF[19]) of the PPSF at the fiber input. At the output end of the PPSF, fiber-pigtailed pump suppression filters reduce the pump power by $>90 \mathrm{~dB}$, at an insertion loss of $6 \mathrm{~dB}$ for the down-converted light. The biphotons then pass through an interferometer which is composed of a polarizing beam splitter, an inline controllable delay line (General Photonics Inc. MDL-001), a polarization controller, and a 50:50 beam splitter. The coincidence rates are depicted in Fig.2(b). An HOMI dip of $26.6 \pm 1.3 \mathrm{fs}$ in width and $83.2 \pm 0.3 \%$ in visibility is obtained from the fitting curve[25] by minimizing the error with respect to the interference pattern. Note that, the interference visibility is limited by the non-optimal components in the interferometer which are designed to operate at $1550 \mathrm{~nm}$ instead of the telecom O-band. Yet the dip visibility still well exceeds the classical limit. The dip width indicates that the bandwidth of biphotons is 138 $\mathrm{nm}$ if the biphotons are transform-limited. The dip width agrees well with our spectral measurement, which is expected because of the low dispersion in PPSF.

Another important and unique feature of the PPSF biphoton source is that it is capable of generating polarization entanglement over much of its bandwidth without any birefringence compensation. The polarization entanglement at various wavelengths of the emission band is measured with the quantum state tomography (QST)[26] experimental setup shown in Fig.3(a). To perform QST at different conjugated wavelength pairs $\lambda_{s}$ and $\lambda_{i}$, three different CWDM sets are used as wavelength splitters. The CWDMs of $17 \mathrm{~nm}$ bandwidth in each set were cascaded such that conjugated wavelengths near the center wavelengths (shown in Table.1) would be transmitted. The biphoton polarization states are analyzed by two polarization analyzers (PAs, Hewlett Packard $8169 A$ ). Each PA includes one polarizer and two waveplates. The commercial PAs were designed for $1550 \mathrm{~nm}$ and therefore the retardances of the waveplates were carefully calibrated for the transmission wavelengths of the CWDMs at telecom O-band.

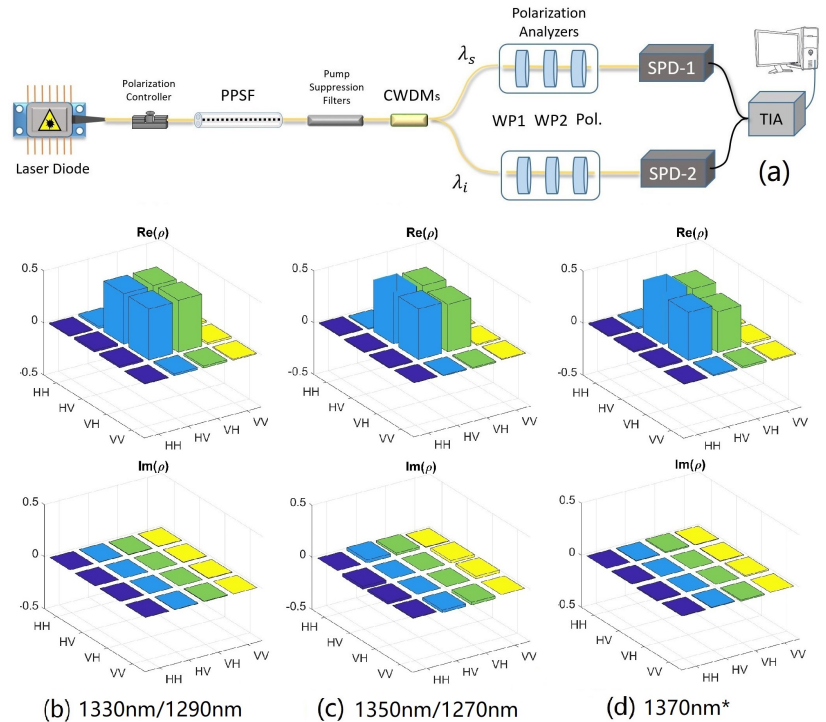

Fig. 3. (a) Experimental setup for polarization state tomography. CWDMs, coarse wavelength division multiplexers; WP, waveplate; Pol., polarizer. (b)-(d) The real and imaginary part of the density matrices of the biphoton states that are measured using CWDMs centered at (b) $1330 \mathrm{~nm}$ and $1290 \mathrm{~nm}$ (c) $1350 \mathrm{~nm}$ and $1270 \mathrm{~nm}$; (d) $1370 \mathrm{~nm}$.

The results of the QST are summarized in Table.1, which shows the concurrences (without subtracting accidentals) of the biphoton states of each wavelength pair. The density matrices of the biphotons states are shown in Fig.3(b)-(d). Neither the measurement apparatus nor the optical components of the source are optimal (they are all designed for 1550nm except for the PPSF), yet we are able to obtain concurrences of more than 0.91 in the biphoton emission band, even when the biphotons are more than $120 \mathrm{~nm}$ apart.

In summary, we have demonstrated a broadband polarization-entangled photon-pair source in PPSF by exploiting type-II SPDC at telecom O-band. The cw-pumped PPSF source emits highly time-correlated and high quality polarization-entangled biphotons of more than $130 \mathrm{~nm}$ bandwidth. The concurrence of polarization entanglement is measured to be greater than 0.91 even when the signal-idler 
Table 1. The experimental results of the quantum state tomography: the concurrence[17], and the fidelity to a maximally polarization-entangled biphoton state that are measured using three CWDM sets at different wavelengths. Each of the CWDM filters has a top-hat transmission profile and a $3 \mathrm{~dB}$ bandwidth of $17 \mathrm{~nm}$.

\begin{tabular}{|l|l|l|}
\hline Wavelengths $\lambda_{s}, \lambda_{i}$ & Concurrence & Fidelity \\
\hline $1330 \mathrm{~nm}, 1290 \mathrm{~nm}$ & $0.9570 \pm 0.0075$ & $0.9779 \pm 0.0037$ \\
\hline $1350 \mathrm{~nm}, 1270 \mathrm{~nm}$ & $0.9372 \pm 0.0124$ & $0.9658 \pm 0.0063$ \\
\hline $1370 \mathrm{~nm}^{*}$ & $0.9110 \pm 0.0131$ & $0.9546 \pm 0.0065$ \\
\hline
\end{tabular}

* CWDM centered on 1250nm is not available to us. Instead of using a second CWDM at $1250 \mathrm{~nm}$, we used the refection port of the $1370 \mathrm{~nm}$ CWDM, which has a broad reflection spectrum, for the idler's measurement.

is greater than $120 \mathrm{~nm}$ apart. The O-band PPSF source can be further optimized. For instance, the PPSF in the context of this letter has a non-zero chromatic dispersion $\left(\left|k_{2}\right| \sim 2 p s^{2} / \mathrm{km}\right)$ at the current QPM wavelength. The biphoton emission spectrum can be potentially broadened to be more than a few hundreds of nanometers if the QPM wavelength is tuned to be closer to the zero-dispersion wavelength of the PPSF. Moreover, using better components such as pump suppression filters and beam splitters with lower loss and better performance will surely result in higher entanglement quality as well.

This simple fiber-based source is compatible with existing fiber infrastructure. Operating near the zero-dispersion wavelength of silica fiber, the time-correlated biphotons generated in this source are expected to be robust against chromatic dispersion in long-distance quantum fiber communication. Through further development, we anticipate that the source can be used in quantum applications such as wavelength-multiplexed quantum communication[14, 27, 28] and high precision quantum sensing $[4,5,7,29-31]$.

Funding. Natural Sciences and Engineering Research Council of Canada (RGPIN-2019-07019, RGPAS-2019-00113, CREATE484907-16), Mitacs Globalink Research Award-Abroad, and US Army Award W911NF20-2-0242. The U.S. Government has a copyright license in this work pursuant to a Cooperative Research and Development Agreement with University of Toronto

Disclosures. The authors declare no conflicts of interest.

\section{REFERENCES}

1. S. Slussarenko and G. J. Pryde, Appl. Phys. Rev. 6, 041303 (2019).

2. P. G. Kwiat, K. Mattle, H. Weinfurter, A. Zeilinger, A. V. Sergienko, and Y. Shih, Phys. Rev. Lett. 75, 4337 (1995).

3. Y. Zhang, F. S. Roux, T. Konrad, M. Agnew, J. Leach, and A. Forbes, Sci. advances 2, e1501165 (2016).

4. F. Kaiser, P. Vergyris, D. Aktas, C. Babin, L. Labonté, and S. Tanzilli, Light. Sci. \& Appl. 7, 17163 (2018).

5. M. B. Nasr, B. E. A. Saleh, A. V. Sergienko, and M. C. Teich, Phys. Rev. Lett. 91, 083601 (2003).

6. H. C. Lim, A. Yoshizawa, H. Tsuchida, and K. Kikuchi, Opt. express 16 16052 (2008).

7. A. Valencia, G. Scarcelli, and Y. Shih, Appl. Phys. Lett. 85, 2655 (2004).

8. J. A. Grieve, Y. Shi, H. S. Poh, C. Kurtsiefer, and A. Ling, Appl. Phys. Lett. 114, 131106 (2019).

9. A. Martin, V. Cristofori, P. Aboussouan, H. Herrmann, W. Sohler, D. Ostrowsky, O. Alibart, and S. Tanzilli, Opt. Express 17, 1033 (2009).

10. T. Zhong, F. N. C. Wong, T. D. Roberts, and P. Battle, Opt. Express 17, 12019 (2009).
11. M. T. Liu, Y. Huang, W. Wang, and H. C. Lim, "Broadband quantumcorrelated photon-pairs in the o-band generated from a dispersionengineered silicon waveguide," in 2013 Conference on Lasers ElectroOptics Europe International Quantum Electronics Conference CLEO EUROPE/IQEC, (2013), pp. 1-1.

12. M. B. Nasr, S. Carrasco, B. E. A. Saleh, A. V. Sergienko, M. C. Teich, J. P. Torres, L. Torner, D. S. Hum, and M. M. Fejer, Phys. Rev. Lett. 100, 183601 (2008).

13. M. C. Booth, B. E. Saleh, and M. C. Teich, Opt. Commun. 284, 2542 (2011).

14. S. K. Joshi, D. Aktas, S. Wengerowsky, M. Lončarić, S. P. Neumann, B. Liu, T. Scheidl, G. C. Lorenzo, Ž. Samec, L. Kling, A. Qiu, M. Razavi, M. Stipčević, J. G. Rarity, and R. Ursin, Sci. Adv. 6, eaba0959 (2020).

15. T. Zhong, X. Hu, F. N. C. Wong, K. K. Berggren, T. D. Roberts, and P. Battle, Opt. Lett. 35, 1392 (2010).

16. T. Kim, M. Fiorentino, and F. N. C. Wong, Phys. Rev. A 73, 012316 (2006).

17. W. K. Wootters, Phys. Rev. Lett. 80, 2245 (1998).

18. A. Christ, A. Fedrizzi, H. Hübel, T. Jennewein, and C. Silberhorn, Single-Photon Generation and Detection: Chapter 11. Parametric Down-Conversion, vol. 45 (Elsevier Inc. Chapters, 2013).

19. E. Y. Zhu, Z. Tang, L. Qian, L. G. Helt, M. Liscidini, J. E. Sipe, C. Corbari, A. Canagasabey, M. Ibsen, and P. G. Kazansky, Phys. Rev. Lett. 108, 213902 (2012).

20. C. Chen, E. Y. Zhu, A. Riazi, A. V. Gladyshev, C. Corbari, M. Ibsen, P. G. Kazansky, and L. Qian, Opt. Express 25, 22667 (2017).

21. E. Y. Zhu, Z. Tang, L. Qian, L. G. Helt, M. Liscidini, J. E. Sipe, C. Corbari, A. Canagasabey, M. Ibsen, and P. G. Kazansky, Opt. Lett. 38, 4397 (2013).

22. Corning, Corning SMF-28 Optical Fibers.

23. A. Canagasabey, C. Corbari, A. V. Gladyshev, F. Liegeois, S. Guillemet, Y. Hernandez, M. V. Yashkov, A. Kosolapov, E. M. Dianov, M. Ibsen, and P. G. Kazansky, Opt. Lett. 34, 2483 (2009).

24. C. K. Hong, Z. Y. Ou, and L. Mandel, Phys. Rev. Lett. 59, 2044 (1987).

25. A. M. Brańczyk, "Hong-ou-mandel interference," (2017).

26. D. F. V. James, P. G. Kwiat, W. J. Munro, and A. G. White, Phys. Rev. A 64, 052312 (2001).

27. S. Wengerowsky, S. K. Joshi, F. Steinlechner, H. Hübel, and R. Ursin, Nature 564, 225 (2018).

28. E. Y. Zhu, C. Corbari, A. Gladyshev, P. G. Kazansky, H.-K. Lo, and L. Qian, J. Opt. Soc. Am. B 36, B1 (2019).

29. A. Riazi, C. Chen, E. Y. Zhu, A. V. Gladyshev, P. G. Kazansky, J. E. Sipe, and L. Qian, npj Quantum Inf. 5, 1 (2019).

30. D. A. Kalashnikov, A. V. Paterova, S. P. Kulik, and L. A. Krivitsky, Nat. Photonics 10, 98 (2016).

31. A. Riazi, E. Y. Zhu, C. Chen, A. V. Gladyshev, P. G. Kazansky, and L. Qian, Opt. letters 44, 1484 (2019). 